TRABAJO EN PROGRESO

\title{
Aplicación móvil para recolección de datos de árboles usando crowdsourcing
}

\author{
Erick Franco-Gaona, Maria Susana Avila-Garcia
}

Publicado: 31 Octubre 2018

\begin{abstract}
Resumen
En este artículo se presenta el trabajo en progreso del diseño de una aplicación móvil para asistir la recolección de la población de árboles. La idea de recolectar información sobre la población de árboles inició como un proyecto local para satisfacer las necesidades del Departamento de Estudios Multidisciplinarios de reportar información sobre su población de árboles. La recolección inicial de fotografías y datos de los árboles se llevó acabo usando cámaras fotográficas y registros manuales sobre la posición de estos en un mapa croquis. Mientras esta solución cumplió con las expectativas en un inicio, la necesidad de mantener actualizada la información llevó a la propuesta de desarrollar una aplicación móvil que permita recolectar esta información de una manera más sencilla y con la ayuda de la comunidad estudiantil. En este trabajo se presentan los resultados preliminares del diseño y desarrollo de dicho proyecto que incluyen la definición de requisitos de la aplicación, el diseño y desarrollo de un primer prototipo y se plantea la metodología a seguir para su evaluación.
\end{abstract}

Palabras clave: Mapa interactivo; Aplicación móvil; Técnicas de recolección; Multitáctil; Evaluación de Usabilidad..

\section{Introducción}

Actualmente vivimos en un mundo globalizado donde la tecnología se usa para apoyar procesos en diferentes áreas como la educación ambiental y la concientización del cuidado del medio ambiente sobre todo en niños y jóvenes que son los más apegados con la tecnología. Según el INEGI en [9] los resultados de la encuesta nacional sobre disponibilidad y uso de tecnologías de la información reporta que en México hasta en el año 2017 el 72.2\% de la población mayor a los 6 años contaba con un teléfono inteligente y de una manera exponencial se espera en los próximos años que este porcentaje se incremente significativamente incluso en edades aún más tempranas. Por ello, es importante impulsar la educación ambiental usando tecnologías móviles para la divulgación de este tipo de temas y promover la participación de la juventud en proyectos de impacto ecológico y concientizar de una manera más atractiva para las personas.

\footnotetext{
Franco-Gaona, E., Avila-Garcia, MS.

Departamento de Estudios Multidisciplinarios

Universidad de Guanajuato

Yuriria, México.

Email: e.francogaona@ugto.mx, susana.avila@ugto.mx
}

Este proyecto tiene como antecedentes el trabajo reportado en [1] donde se presentó como primer prototipo un mapa interactivo diseñado en la herramienta Intuiface composer que permite el diseño rápido de aplicaciones multitáctiles por medio de configuración de componentes sin la necesidad de agregar una sola línea de código. El mapa multitáctil que fue presentado a la comunidad estudiantil en el Departamento de Estudios Multidisciplinarios (DEM) de la Universidad de Guanajuato en la Sede de Yuriria con el objetivo de promover la educación ambiental y la cultura del árbol. Como se muestra en la Figura 1, el mapa presenta la ubicación relativa de los árboles, definida por aproximación, además de las fotografías recolectadas usando una cámara fotográfica. La retroalimentación obtenida por parte de los estudiantes y de las personas encargadas de reportar información a la Procuraduría Ambiental y de Ordenamiento Territorial fue positiva indicando que encontraban valor en la aplicación y como parte de la retroalimentación se identificaron oportunidades de mejora.

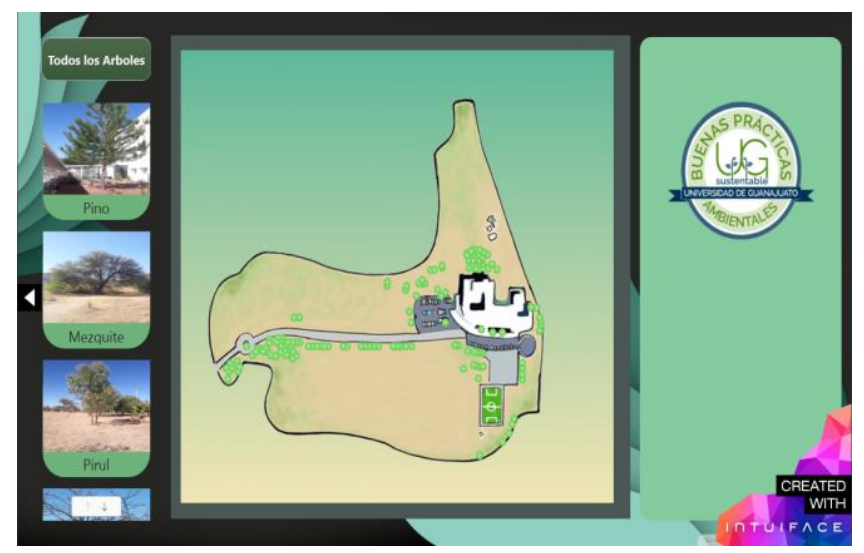

Figura 1. Pantalla de captura del mapa interactivo reportado en [1].

Una de las características de valor del mapa interactivo fue el proporcionar de manera indirecta un inventario de la población de árboles de la institución, proporcionando un valor agregado a la necesidad básica de contar información solo de los árboles reforestados. Tener un inventario de árboles ayuda a la institución a tener un mejor control de las estadísticas requeridas con fines de certificación ambiental. Contabilizar los árboles ayuda a obtener el índice de huella verde de la institución, estadística que no se tenía considerada de manera inicial. 
Otra oportunidad de mejora para el proyecto se refiere a eficientar la manera de recolectar información de los árboles para mantener la base de datos actualizada y registrar la ubicación de los árboles con su posición geográfica. Inicialmente, como parte del proceso de captura de información para el mapa interactivo de árboles reportado en [1], parte del equipo de trabajo debía ir con una cámara fotográfica hasta la ubicación de los árboles para obtener la fotografía y en un mapa croquis en papel marcar la zona donde fue tomada, posteriormente se consultaban especialistas en el área de ingeniería ambiental, y fuentes de la SEMARNAT [13] para su clasificación. Como se puede observar este proceso es un poco tardado por lo que se plantea este proyecto como nueva forma de recolección de datos para mantener actualizado el inventario de árboles. El objetivo a largo plazo es tener un sistema de información de población de árboles usando diferentes interfaces para la recolección de datos y la visualización de información.

El objetivo es también impulsar a la comunidad estudiantil a tomar un rol activo cultivando así una cultura ecológica y haciendo conciencia de la importancia e impacto que los árboles tienen en nuestra comunidad. La captura fotografías con una aplicación móvil, permite utilizar los recursos disponibles en el dispositivo para capturar la localización del punto donde la fotografía fue tomada, y diseñando opciones que permitan clasificarlo o etiquetarlo como no clasificado para llevar un mejor control de la consistencia e integridad de los datos con los que se cuenta.

En [3] se presenta la prueba de concepto de una aplicación móvil desarrollada en App Inventor para tomar fotografías de los árboles. Aunque la aplicación fue exitosa como prueba de concepto, no es viable continuar con el desarrollo de esta en App Inventor ya que esta herramienta no ofrece la flexibilidad de desarrollos más especializados.

El entorno de desarrollo que se recomienda para nuestro diseño está basado en el sistema operativo Android por la cantidad de dispositivos a nivel mundial que lo usan. Como lo demuestra la empresa Gartner en un estudio realizado se muestra en [5] que el sistema operativo más utilizado es Android con un $84.1 \%$ del mercado. De los entornos de programación integrados como programa de aplicación (IDE) más utilizados para el desarrollo en Android se encuentran Eclipse que durante varios años fue la mejor alternativa según un blog publicado en la página de IBM para desarrollo móvil, con el cual incluso introducen a las aplicaciones móviles en [4]. También se cuenta con Xamarin la alternativa que genera Microsoft como IDE multiplataforma que puede trabajar tanto en iOS como en Android con un mismo código y usa el lenguaje de programación C\#. Sin embargo, actualmente el más utilizado es Android Studio por la gran cantidad de documentación que existe gracias al respaldo de Google y este IDE es el sucesor del Eclipse como el recomendado por Google.

En la búsqueda de mayor flexibilidad, en este trabajo se presenta el trabajo en progreso de la propuesta de diseño y desarrollo una aplicación móvil en Android que permita la recolección de datos de los árboles que incluye, pero no se limita a fotografías y sus metadatos para recuperar la ubicación de donde fue tomada la fotografía. Se muestran resultados preliminares de la definición de requisitos de la aplicación, el diseño y desarrollo de un primer prototipo, y finalmente, se define la metodología a seguir para su evaluación.

\section{Estado del arte}

Existen varias propuestas en el internet de aplicaciones que asisten la creación de inventarios de árboles. Por ejemplo, la aplicación Tree Survey App, descrita en [2] es una aplicación móvil usada para capturar información de árboles que incluye la identificación de la persona que recolectó la información, el tipo de árbol, el tamaño del árbol, la edad, especificación de si es un árbol frutal, características de los elementos que se encuentran a su alrededor, algún defecto que pueda tener, etc. Esta aplicación es usada por consultorías Arboriculturales y autoridades locales para ayudar a manejar los árboles. Otro proyecto que ofrece la funcionalidad de crear mapas de árboles es OpenTreeMap descrita en [12] usando dispositivos móviles como teléfonos y tabletas para recolectar y actualizar la información. Al momento de recolectar información sobre los árboles se agrega información sobre la localización del árbol, así como el número de troncos del árbol, incluyendo el diámetro o la circunferencia de estos, etc. Otras iniciativas como [https://urbanforestmap.org/], están enfocadas a recolectar información de los árboles en la ciudad de San Francisco en Estados Unidos, en esta aplicación se puede apreciar de manera cuantitativa los beneficios ecológicos de la población de árboles registrada en la ciudad, la energía conservada, la cantidad de agua de lluvias filtrada, calidad del aire mejorada, cantidad de dióxido de carbono eliminado al año, etc. En [10] se propone un trabajo para la gestión de árboles basado en geolocalización como herramienta para llevar un control en la poda y tala de árboles que una empresa en Colombia realiza sobre vegetación que se encuentra en proximidad a redes eléctricas. Por otro lado, en [11] se presenta una aplicación móvil para clasificar hojas de árbol según a la especie que pertenezcan haciendo uso de la visión por computadora y la librería OpenCV. El objetivo es indicar a que árbol pertenece una hoja cualquiera para tener una clasificación bien ordenada guardada en una base de datos.

Finalmente, en [3] se muestra un trabajo previo al reportado en el presente artículo donde se presenta la primera versión de la aplicación desarrollada en App Inventor una plataforma de diseño rápido de aplicaciones y tiene como objetivo mostrar las ubicaciones geográficas de los árboles, así como información de estos. En la Figura 2 se presentan screenshots de este prototipo.

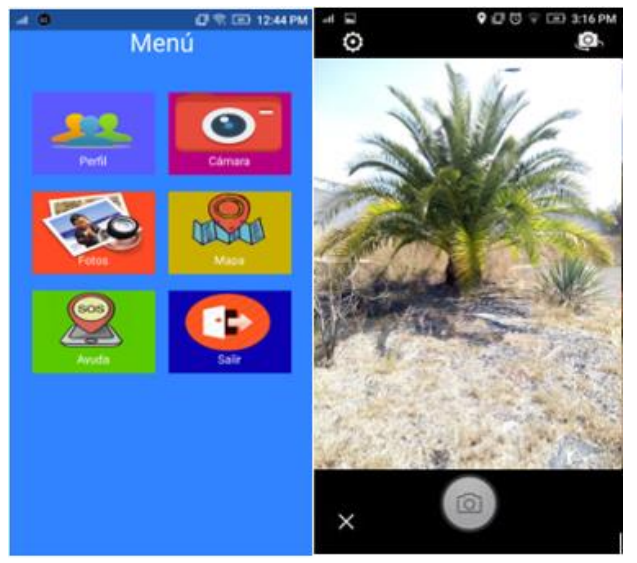

Figura 2. Prototipo desarrollado en AppInventor

\section{Metodología}

La metodología de desarrollo que se utiliza en esta aplicación es una basada en el desarrollo ágil que se conoce como Mobile-D que según [14] es muy recomendada para desarrollo móvil en la actualidad pues se basa en Extreme programming y metodologías 
relativamente nuevas para el desarrollo rápido y estable del software y sus fases son las siguientes: exploración, inicialización, desarrollo, estabilización y pruebas del sistema, esto se realiza en ciclo para mejorar cada entrega como producto de valor.

Se utilizan algunos estándares para desarrollo de software como el ISO IEEE 15288 reportado en [7] que contiene los procesos técnicos utilizados para el desarrollo de software y el ISO IEEE 29148 reportado en [6] que habla sobre la ingeniería de requisitos y las recomendaciones para la especificación de requisitos. De la lista de requisitos los listados a continuación son los fundamentales para el primer producto de valor de la aplicación.

Tabla 1. Tabla de requisitos iniciales de la aplicación.

\begin{tabular}{|l|l|}
\hline \multicolumn{1}{|c|}{ ID } & \multicolumn{1}{|c|}{ Requisito } \\
\hline 1 & $\begin{array}{l}\text { La aplicación debe tener permiso de uso de la cámara del } \\
\text { dispositivo móvil al momento de instalación. }\end{array}$ \\
\hline 2 & $\begin{array}{l}\text { La aplicación debe pedir permiso para el uso del GPS del } \\
\text { dispositivo móvil al momento de instalación. }\end{array}$ \\
\hline 3 & $\begin{array}{l}\text { La aplicación debe crear un folder XXX al momento de } \\
\text { instalación que deberá ser utilizado para guardar la } \\
\text { información generada por la aplicación. }\end{array}$ \\
\hline 4 & $\begin{array}{l}\text { La aplicación debe tomar una fotografía y guardarla en el } \\
\text { folder creado en Requisito \#2 en el dispositivo. }\end{array}$ \\
\hline 5 & $\begin{array}{l}\text { La aplicación debe extraer y guardar los metadatos de las } \\
\text { fotografías. }\end{array}$ \\
\hline 5.2 & $\begin{array}{l}\text { La aplicación debe extraer el metadato de fecha de creación } \\
\text { de la fotografía }\end{array}$ \\
\hline 5.3 & $\begin{array}{l}\text { La aplicación debe extraer el metadato de latitud de la } \\
\text { ubicación GPS de la fotografía. }\end{array}$ \\
\hline 5.4 & $\begin{array}{l}\text { La aplicación debe extraer el metadato de longitud de la } \\
\text { ubicación GPS de la fotografía. }\end{array}$ \\
\hline 5.5 & $\begin{array}{l}\text { La aplicación debe definir el formato de nombre de la } \\
\text { fotografía con la fecha y hora del sistema }\end{array}$ \\
\hline 6 & $\begin{array}{l}\text { La aplicación debe extraer el metadato de nombre de la } \\
\text { metadatos guardados. }\end{array}$ \\
\hline
\end{tabular}

\section{Resultados preliminares y discusión}

El prototipo desarrollado cumple con los requisitos establecidos en la Tabla 1. La aplicación solicita permiso de uso de la cámara y de uso del GPS al tomar fotografías concediendo los correspondientes permisos por seguridad. Además, la aplicación guarda las imágenes capturadas en el dispositivo en una pequeña base de datos en SQLite que provee el mismo IDE para posteriormente mostrar esa información. El diseño de esta versión puede verse en la Figura 3.

El caso de uso para tomar una fotografía inicia al abrir la aplicación, donde el usuario se encuentra con la pantalla presentada en la Figura 3 (izquierda). El usuario puede tomar la fotografía al tocar el botón fotografía para acceder a la cámara del teléfono, cabe mencionar que la aplicación enciende la ubicación automáticamente. Al entrar en modo cámara se espera la captura de fotografía y al momento de tomarla se guarda en el dispositivo y se comienza a hacer el escaneo de metadatos una vez obtenidos se almacenar en la base de datos interna y se visualiza la fotografía tomada en la aplicación., se genera una opción para observar los metadatos guardados
La aplicación ha sido probada en un ambiente controlado por los desarrolladores. Al tomar fotografías los metadatos se extraen con éxito de la fotografía como se esperaba. La ubicación debe encenderse tanto en el teléfono como en la cámara.

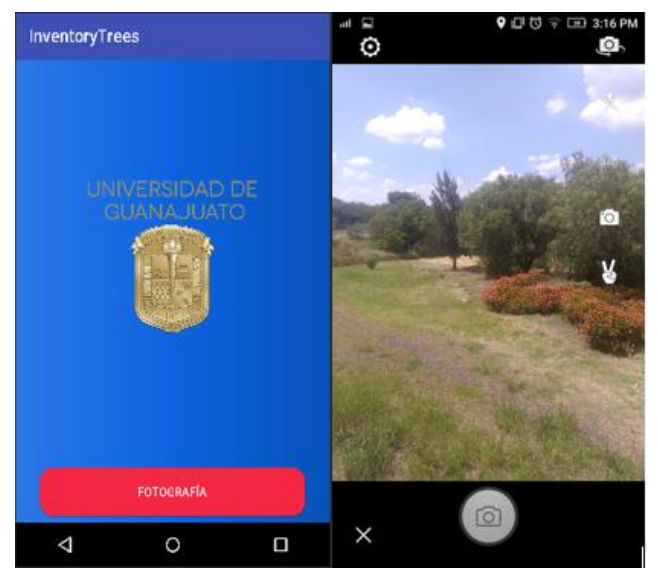

Figura 3. Prototipo desarrollado en Java usando Android Studio.

\section{Conclusiones y trabajo futuro}

Este proyecto tiene un gran alcance pues el trabajo en conjunto con un mapa interactivo y multitáctil enriquece la información ecológica que en este caso se trata de compartir. Este proyecto no solo sirve para una certificación si no para concientizar el cuidado de nuestros arboles pues se pretende que se generen estadísticas más concisas y fáciles de observar para cualquier tipo de usuario. Tratamos de aprovechar el auge de las nuevas tecnologías, así como el tipo de desarrollo interactivo para hacer que las personas en general hagan una reflexión sobre el cuidado del medio ambiente. Además, se busca que la institución tenga información sobre el impacto de los beneficios ecológicos de su población de árboles.

Este trabajo ofrece una gran ventaja sobre la manera anterior de recolectar información de los árboles. Como parte del trabajo futuro se tiene contemplado el continuar el desarrollo de esta aplicación con una metodología SCRUM. La aplicación se ha puesto a prueba con un conjunto de usuarios en un ambiente controlado que usan diferentes versiones de Andriod, por lo que se espera tener una evaluación más completa de la aplicación, y se realizará un focus group para recolectar la retroalimentación del uso de la aplicación. Los siguientes desarrollos están enfocados en la identificación del árbol dentro de la fotografía, así como su clasificación. Se esperan mejoras significativas al realizar la clasificación. Inicialmente se realizará la identificación del árbol de manera manual, y en siguientes sprints se comenzarán a probar técnicas de visión por computadora para identificar los árboles, especializando la identificación y clasificación de estos con el paso del tiempo.

Las evaluaciones de las liberaciones de la aplicación tomarán en cuenta aspectos de la calidad de software espcificadas en el ISO/IEC 25010:2011 [8] tomando en consideración las siguientes características: funcionalidad, confiabilidad, usabilidad, eficiencia, mantenibilidad, y portabilidad. Los aspectos de usabilidad que estaremos monitoreando en cada evaluación son: efectividad, eficiencia, seguridad, utilidad, facilidad de aprendizaje de uso, y satisfacción del usuario.

Se seguirá la metodología de trabajo de diseño interactivo siguiendo un diseño centrado en el usuario, definiendo usuarios 
claves que nos den retroalimentación a lo largo del desarrollo del proyecto.

La arquitectura del proyecto estará basada en servicios web con una variedad de interfaces para diferentes dispositivos. Además, se tiene contemplado configurar la aplicación para trabajar en dos modos principales: en línea y fuera de línea, ya que en algunas zonas la conexión a internet puede no estar disponible. Al momento en que el usuario se conecte nuevamente en línea podrá dar la instrucción de enviar los datos recolectos al servidor.

Dado que la aplicación tiene contemplada la participación ciudadana, se deben considerar mecanismos para reconocer las aportaciones de los participantes dando el debido crédito por las aportaciones realizadas que pueden ir desde la captura de fotos, identificación y clasificación de árboles, mientras se desarrollan técnicas que vayan asistiendo estos procesos de manera más automática.

La clasificación de los árboles puede ser difícil si no se tiene conocimiento sobre éstas, por lo que se tiene contemplada la participación, no solo de los estudiantes y de la población en general, sino de personas que estén interesadas en estos temas y puedan aportar y ayudar a la clasificación de los árboles en caso de que los usuarios no puedan identificarlo al momento de tomar la fotografía.

\section{Agradecimientos}

Los autores agradecen a los stakeholders de este proyecto por proporcionar su tiempo en el análisis de requisitos. Los autores agradecen a los stakeholders de este proyecto por proporcionar su tiempo en el análisis de requisitos. Este trabajo está soportado por el proyecto SEP-PRODEP UGTO-PTC-510.

\section{Referencias}

[1] B. Q. Guzmán, M. S. Á. García, F. A. Á. García, M. Bianchetti, and E. F. Gaona, "Diseño De Mapa Interactivo Y Multitáctil De Supervivencia De Árboles.," Pist. Educ., vol. 39, no. 127, pp. 426-436, 2018.

[2] E. James, "Providing Bespoke Data Capture Solutions" 2017. [Online]. Available: http://www.gissolutions.co.uk/Mobile\%20app\%20for\%20tree\%20surveys.h tm.

[3] F. G. Erick, Á. García, and M. Susana, "Diseño de Aplicación Móvil para Inventario de Árboles,” no. 1, pp. 15.

[4] Frank Ableson, "Desarrolle aplicaciones Android con Eclipse," 2013. [Online]. Available: https://www.ibm.com/developerworks/ssa/opensource/tutoria 1s/os-eclipse-android/index.html. [Accessed: 22-Aug-2018].
[5] Gartner Inc., "Gartner Says Worldwide Sales of Smartphones Grew 9 Percent in First Quarter of 2017," 2018. [Online]. Available: https://www.gartner.com/en/newsroom/pressreleases/2017-05-23-gartner-says-worldwide-sales-ofsmartphones-grew-9-percent-in-first-quarter-of-2017. [Accessed: 07-Jul-2018].

[6] I. Standard, "INTERNATIONAL STANDARD ISO / IEC / IEEE Systems and software engineering - System life cycle processes - Requirements engineering," 2011.

[7] I. Standard, "INTERNATIONAL STANDARD ISO / IEC / IEEE Systems and software engineering — System life cycle processes," vol. 17.

[8] I. Standard, "Systems and software engineering -- Systems and software Quality Requirements and Evaluation (SQuaRE) -- System and software quality models” 2011. [Online]. Available: https://www.iso.org/standard/35733.html.

[9] INEGI, Secretaría de Comunicaciones y Transportes, and Instituto Federal de Telecomunicaciones, "En México 71.3 millones de usuarios de internet y 17.4 millones de hogares con conexión a este servicio: ENDUTIH 2017," 2018. [Online]. Available: http://www.beta.inegi.org.mx/contenidos/saladeprensa/boleti nes/2018/OtrTemEcon/ENDUTIH2018_02.pdf.

[10] J. A. Castrillón Arboleda, B. Silveira Moreno, “CEIBA: Aplicación Móvil para la Gestión de Arbolado Basada en Geolocalización,” 2018.

[11] J. Blanco Alambiaga, "Desarrollo De Una Aplicación Móvil Para Le Detección Y Clasificación De Hojas De Árboles," 2015.

[12] OpenTreeMap, “Open tree map track youy tree care" 2017. [Online]. Available: https://www.opentreemap.org/.

[13] Procuraduría Federal de Protección al Ambiente, "NORMA OFICIAL MEXICANA NOM-059-SEMARNAT-2010," 2016. [Online]. Available: https://www.gob.mx/profepa/documentos/norma-oficialmexicana-nom-059-semarnat-2010. [Accessed: 20-Sep2018].

[14] Universidad Santiago de Cali et al., "Evolution of Mobile Software Development from Platform-Specific to WebBased Multiplatform Paradigm," Rev. Tecnol. | J. Technol., vol. 12 número, no. 4, pp. 181-183, 2013. 